Article

\title{
Applications of Blockchain Technology to Logistics Management in Integrated Casinos and Entertainment
}

\author{
Da-Yin Liao $^{1, *}$ and Xuehong Wang ${ }^{2}$ \\ 1 Straight \& Up Intelligent Innovation Group Co., San Jose, CA 95113, USA \\ 2 Chinese Academy of Fiscal Sciences, Ministry of Finance of the People's Republic of China, Beijing 100142, \\ China; wxh1241001@163.com \\ * Correspondence: eliao@necksoft.com; Tel.: +1-408-332-0604
}

Received: 21 September 2018; Accepted: 22 November 2018; Published: 27 November 2018

\begin{abstract}
The gaming industry has evolved into a multi-functional smart city that combines integrated casinos and entertainment (ICE). ICE logistics involve supply chains with various stages in geographically-distributed locations and with limited and complex storage and warehouses. Challenges are to leverage demands, traffic, and storage allocation in ICE logistics. The decentralized structure of blockchain technology allows all parties to participate in ICE logistics. Its cryptography-based, immutable nature gives the assurance of security. This research deals with the design and application of blockchains in ICE logistics. We first adopt a Concentric Value Circles (CVC) model to identify the requirements and business opportunities that use blockchain technology in ICE logistics. We develop an open, automated, and transparent platform, TransICE, which utilizes the feature of smart contracts in blockchain technology and adopts a decentralized model, Hawk, where no financial transactions are stored on the blockchain to hold privacy of transactions publicly. Two cases, (1) the Shipment Pricing and Scheduling process and (2) the Pickup, Shipping and Delivery process in TransICE, are studied to illustrate the applications and feasibility of the proposed TransICE platform and the developed smart contracts of the Hawk model.
\end{abstract}

Keywords: blockchain; integrated casinos and entertainment; supply chain; logistics; gaming; concentric value circles; Ethereum; Hawk; smart contracts; distributed ledgers; transparency; privacy; trustless

\section{Introduction}

Modern public administration has evolved into public service. Rather than to attempt to control societies, the new role of a government has changed to encourage citizens to articulate and achieve their shared interests [1]. As a focus of the governments' regulatory spotlight, the gaming industry is also changing profoundly, creating major new sources of revenue and value beyond casino gambling and lodging. The contemporary gaming industry has evolved into a multi-functional city that combines casinos and attraction tourism (CAT); integrated resorts (IR) and hotels; restaurants; retail shopping; theaters; spa, fashion and beauty; live entertainment; sports and racing events; meetings, incentives, conventions and exhibitions (MICE); and theme parks. Integrated Casinos and Entertainment (ICE) is more than just gaming, but has the potential for comprehensive tourist promotion. ICE would boost the array of tourist attractions and facilities. Development and operations of an ICE project in a city provides a long-term competitive advantage for the city as a tourism destination. ICE serves as a way to gain competitiveness for a city in the tourism industry, and has spillover effects on other industries in the city. To promote sustainability, an ICE city not only provides infrastructure and services that 
support tourism, leisure and entertainment, but also seeks to improve the quality of infrastructure and services. A city of ICE is a smart city that demands a clean and smart environment, smart governance, smart logistics, smart transportation, smart technology, and smart health to control the flow of energy, materials, logistics and traffic in the ICE area. ICE that is able to integrate its physical and digital operations will change the game.

The ICE industry is strongly based on service - the very experience that will attract visitors and tourists to the area. As the development of ICE is based on planning for so many visitors, an array of food and beverage (F\&B) offerings are needed as complementary amenities. The breadth and depth of restaurants and lounges create an even greater magnet effect that can enhance the overall appeal of a venue, and allow for even more facilities to be added. Other non-gaming ICE functions include cinemas, showrooms, nightclubs, golf courses, spas, wild animal exhibition areas, art galleries, amusement parks, and retail shopping malls. Gaming, F\&B, lodging, and entertainment are all service-based. And ICE operations reflect the importance of quality of service. Major stakeholders in the ICE value chain [2] include casino service delivery, physical environment, F\&B suppliers, amenities vendors, technology providers, and supervisory agencies [3]. Potential benefits of new technologies for the ICE value chain are listed as below [4]:

- Reduced costs of overall transactions and information and communications infrastructure.

- Irrevocable and tamper-resistant transactions.

- Ability to store and define ownership of any tangible or intangible asset.

- Reduction of systemic risks.

- Consensus in a variety of transactions.

- Increased accuracy of trading information to reduce settlement risk.

- Near-instantaneous clearing and settlement.

- Improved security and efficiency of transactions.

- Enabling effective inspection and auditing processes by participants, supervisors, and regulators.

Logistics, as defined in [5], is a framework to create a plan for the flow of products and information through a business. Upon this framework, supply chain management (SCM) builds linkage and coordinates among players along the logistic pipeline, from suppliers to customers. Therefore, logistics management is an integral part of SCM, and involves several participating parties to integrate the movement of goods, services, information, and capital, right from the source of raw material to its consumers. Establishing communication and information exchange between all involved parties is complex and problematic in logistics processes, especially if new technology is used. The goal of a logistics process is to provide effective management via smart allocation and the scheduling of products, quality, time, place and cost, from suppliers to customers. Activities involved in logistics are usually classified as inbound-e.g., procurement of material, handling, storage and transportation, and outbound - e.g., collection, maintenance, and distribution or delivery to the end consumer. The development of logistics depends on innovations implemented in the industry, new technologies, as well as economic, political, social, and environmental considerations.

The definitions of logistics have been evolving over time as the functions and areas of interest change. Over the past two decades, the intensive use of information and communication technologies (ICT) in industry has increased the operational efficiency and competitiveness in logistics. However, along with the adoption of new technologies, new challenges arise and become overwhelmed. Customers are demanding more and more personalized products and services which impose high levels of complexity and difficulties for both inbound and outbound logistics. The emerging challenges in logistics include the needs for (1) transparency and visibility in logistics; (2) integrity control of logistics, with the right product at right time, place, quantity, quality, and cost; (3) dynamic reconfigurability of logistics by reconfiguring service level agreements (SLAs) with material suppliers and contract manufacturers; and (4) lean, agile, resilient and green logistics [6]. Logistics 4.0 [7,8], also known as Smart Logistics, addresses these challenges and aims to provide Smart Services 
and Smart Products with more intelligence in a logistics system, rather than through automating logistics processes. In addition to the well-established applications such as Enterprise Resource Planning (ERP), Supply Chain Management (SCM), Warehouse Management (WM), and Fleet Management (FM), the new paradigm of Logistics 4.0 is featured by its intelligent networks based on cyber-physical systems (CPS) which are integrated through wireless embedded sensors, actuators, and control/processing/communication devices. Logistics 4.0 is a connection of technical, technological, and organizational solutions. New applications in Logistics 4.0 include Intelligent Transportation Systems (ITS), driverless vehicles, automated identification, automatic materials handling, machine learning, big data analytics, and data security and transparency, where all processes/devices/humans in the logistics networks can communicate with each other to promote full Logistics 4.0 operations.

ICE boosts logistics across all sectors, from lodging, foods and beverages, entertainment, transportation, banking, to government and healthcare. In an ICE city like Marina Bay Sands, Singapore [9], there are hotels with more than two thousand rooms, tens of F\&B outlets and restaurants, dozens of retail shops and malls, casinos with hundreds of tables and thousands of slots, theaters and museums, event plaza, Skypark, and exhibition and meeting space of millions square feet to accommodate more than 45,000 participants. ICE logistics involve supply chains with various stages in geographically-distributed locations and with limited and complex storage and warehouses. Challenges are to leverage demands, traffic, and storage allocation in ICE logistics. In addition, it is difficult to investigate the accountability of anomaly events or incidents associated with the ICE logistics. For example, incidents to food safety and hygiene may take place in the farm-to-fork process-from supplier selection, delivery and receiving, food preparation to food served.

Blockchain technology [10] is receiving attention, with more and more diverse applications, as well as increasing numbers of players involved in its use. It has been gaining a lot of attention from both academic researchers and industrial practitioners to understand the possible applications of blockchain technology to businesses, and particularly, to business models. Blockchain technology involves the combination of databases, transactions, encryption, virtualization, consensuses and distributed system technologies. In blockchains, a distributed data structure, a digital public ledger of data, is created and maintained, and shared among a network of relevant parties. Blockchain technology uses cryptography to allow each participant in a network to maintain the ledger securely, requiring no centralized management of the rules. There are some promising application areas for the deployment of blockchains in the logistics sector, like digital document exchange and the tracking of goods. The blockchain, as an insurer of transparency and security, can improve the flow of goods and information in ICE logistics. With blockchains, public availability gives the opportunity to track goods and services from the place of origin to the end customer. The decentralized structure of blockchains allows all parties to participate in ICE logistics. Its cryptography-based and immutable nature gives the assurance of security. Blockchains have the potential to meet the requirements of information systems in ICE logistics due to their ability to create trust and establish an organization overarching platform to exchange information.

This paper deals with the design and applications of blockchains in integrated casinos and entertainment (ICE) logistics. We adopt a Concentric Value Circles (CVC) model to identify the requirement and business opportunities to adopt blockchain technology in ICE logistics. We analyze critical considerations in building blockchains in ICE logistics, including public availability, decentralized ledgers, and cryptography. The applications and functions of blockchain-based smart contracts [11] are developed to optimize the operations in ICE logistics of receiving turnaround times (TAT), food safety and hygiene, dock scheduling, and offsite consolidation, respectively. We develop an open, automated, and transparent platform, TransICE, which utilizes a decentralized model for smart contracts of blockchains. In the decentralized model, no financial transactions are stored on blockchains, so transactional privacy can be maintained publicly. Two representative cases in ICE logistics-(1) the Shipment Pricing and Scheduling process; and (2) the Pickup, Shipping and Delivery 
process-are analyzed to illustrate the operation flow, applications, and feasibility of the proposed TransICE platform.

The organization of this paper is as follows. Section 1 describes the motivation and identifies the importance and challenges of this research. In Section 2, we review the gaming industry, and provide a short overview of the blockchain technology and how it can disrupt business models. Section 3 presents the operations in ICE logistics and shows that blockchain technology is one of the key drivers of innovation in ICE logistics. A CVC model for blockchain in ICE logistics is developed in Section 4. Section 5 proposes the blockchain-integrated ICE logistics platform that uses smart contracts with the Hawk model. In Section 6, case studies of two salient ICE logistics processes are analyzed to validate how the developed blockchain applications can be used by ICE logistics. Section 7 concludes this paper.

\section{Literature Review}

Modern gaming research can trace its roots back to the 1974 First National Conference on Gambling and Risk-Taking in Las Vegas, NV. In those days, commercial casinos operated only in Nevada, and there was almost no published literature in economics on the gaming industry, except for some research on risk-taking and gambling behavior [12]. The economics of the gaming industry took off when casinos began their expansion outside of Les Vegas and Atlantic City in the early 1990s, and has grown dramatically worldwide. At the same time, Las Vegas has changed her face and image from gambling to casino entertainment, with the openings of mega-casino complexes along the Las Vegas Strip during the 1990s. Non-gaming activities had contributed to nearly 50\% of revenues accrued by the largest 19 casinos in the Las Vegas Strip by 1997, and in 1997, over 80\% of all the profits earned by the more than 200 major casinos in Nevada came from this clustering of mega-casino complexes [13]. By expanding its gaming entertainment industry in 2002, Macau experienced rapid growth from 2002 to 2007. Macau's per capita gross domestic product (GDP) overtook its neighbor Hong Kong, and gaming revenue surpassed that of the Las Vegas Strip in 2006 [14]. The tourism industry in Singapore has continued to grow since two Integrated Resorts-Marina Bay Sands (MBS) and Resorts World Sentosa (RWS)—were launched in 2010 [15]. New mega casino resorts will open in the Philippines, South Korea, Vietnam, and Russia by 2020 [16]. More and more countries are investing millions of dollars in integrated casinos and entertainment (ICE) industries. Relatively few studies have focused on the design and development of logistics management in ICE.

The blockchain is a data structure that creates and maintains an open and distributed ledger shared by a network of independent participants. In the ledger, all transactions between parties are efficiently, verifiably, and permanently recorded [17]. As the core of Bitcoin [18] and other virtual currencies, blockchain technology relies on cryptography to allow any participant on the network to update the open ledger securely, without the need to handle the ledger by a powerful central authority. The decentralized nature of blockchain technology removes the role of central middlemen in handling ledgers, while the information being stored in a blockchain is honest and accurate. A smart contract [19] is a transactional protocol running on top of a blockchain embedded in digital code that executes the terms of a contract where the parties to that contract agree to interact with each other. If and when the pre-defined rules are met, the agreement is automatically enforced. The smart contract code facilitates, verifies, and enforces the negotiation or performance of an agreement or transaction. It is the simplest form of decentralized automation. Smart contracts are stored in transparent, distributed databases, and are shared but protected from distortion, revision, tampering, and deletion. The system is embedded with software that allows applications to be created that operate on the smart contracts principle, that is, stand-alone programs running when certain conditions are met. Each transaction is stored and can be shared with its digital record and signature that could be identified and validated. The immense potential of blockchain technology promotes free transactions and interactions among individuals, organizations, machines, or even computer programs. Blockchain technology is called 
"the second generation of the Internet" [20], and will likely to have a great impact in the near future on the world economy.

Introduced in October 2008, Bitcoin is the first application of blockchain technology. Blockchain is one of the foundational technologies of Bitcoin, and has the potential to create new opportunities for our economic and information systems. While Bitcoin's future could be a mirage, blockchains are real, promising and being used in business. Blockchains are poised to change how people do business by offering trust, similar to how the Internet changed the world by providing greater access to information [21]. In addition to the financial sector, many other industries have showed interests in blockchain technology, and have been exploring use cases or business opportunities in various applications. Applications of blockchains in the built environment can be found in smart energy, smart cities, smart government, smart homes, business models and organization structures, and so on [22]. The potential benefits of blockchains for business are numerous. Blockchain technology can help reduce time for finding information, settling disputes, and verifying transactions. It can decrease costs for overheads and intermediaries. And blockchains can alleviate risks of collusion, tampering, and fraud. As a technology that offers highly efficient data integrity, transparency, security, anti-forgery and anti-tampering, blockchains can be used in various business activities which involve secure data exchange. Blockchain technology provides the ability to decentralize applications for the participation of all parties in ICE logistics. Its cryptography-based, immutable nature assures security and accuracy in ICE logistics. This research deals with the design and application of blockchains for effective logistics management in ICE.

While blockchain technology holds considerable promise to applications in various fields, the most interesting areas for this technology is logistics that are ready for disruption. Logistics, as a planning structure for material, service, and information flows management, typically involves the integration of complicated information flow, transportation, warehousing, and security. Blockchains are one of the greatest potentials for the development of e-logistics [23], which is essentially a complex system comprised of producers, distributors, resellers, carriers, and consumers, with electronic exchange of data via the Internet or dedicated communications networks. As supply chain integration becomes increasingly dynamic, access to customer demand needs to be shared. And deliveries of products and services must be tracked effectively to provide visibility and controls in the supply chain. Blockchain technology is identified as an enabler to achieve disruptive transformation in supply chain integration [24]. Gallay et al. [25] propose a peer-to-peer platform for decentralized logistics to magnify and accelerate the impact offered by the integration of the most advanced technologies in ICT, including blockchain technology, to innovate logistics operations with a fully decentralized, integrated, and trusted platform. Diveco Logistics [26] is a cloud-based logistics platform and also an Enterprise Resource Planning (ERP) system, based on blockchain and Internet of Things (IoT) technologies in the Tangle [27] registry. QUASA [28] is a decentralized logistic platform that creates a transparent system of interactions for all participants, and ensures privacy and security in the supply chain. LogisticsX [29] develops a decentralized application on blockchains for the future of last mile logistics, where more stakeholders are involved than the traditional Hub-and-Spoke distribution model [30], and traceability and transparency in the last mile delivery increases.

Every company should have a business model, and every business model is inherently subject to changes if it is to succeed. Although industrial practitioners have used the term "business model" for years, the topic has received attention by academic researchers only recently [31,32]. As new technologies along with novel innovative business models can shape the competitive advantages for companies, it is important to explore how new technologies, like blockchains, may contribute to innovation and disruption in business models. Nowiński and Kozma [33] identify three crucial ways for blockchain technology to disrupt and change business models. They are (i) authentication of traded goods, (ii) disintermediation, and (iii) reduction of transaction costs. Their study analyzes the possible applications of blockchain technology to business models, as well as the business itself. While blockchain technology may bring promising benefits like transparency, the current adoption 
of blockchains in supply chains is still limited or being developing. Francisco and Swanson [34] use the concepts of the Technology Innovation Adoption to study technology adoption of blockchain for transparency in supply chains. To the best of our knowledge, there is no published work about the analysis of business models for blockchains in the ICE industry. In Section 4, we adopt a Concentric Value Circles (CVC) model [35] for analysis of the potential benefits of blockchains in ICE logistics.

\section{Logistics Management in ICE}

ICE logistics involves management of tourism, hotel/meeting/convention, floor, retail, and sustainability. Figure 1 shows the operations in ICE logistics, ranging from storage, to distribution, to transportation, to fulfill the various and dynamical requirements on FF\&E (furniture, fixtures \& equipment), OS\&C (operating supplies \& consumable), F\&B, apparels, clothing, shoes \& jewels, gifts \& souvenirs, artworks, luxuries, books, CDs (compact disks), electronics, healthcare, and pet supplies for daily necessities in ICE activities. Challenges in ICE logistics can occur in inbound traffic, distributed and limited storage, warehouse complexity, receiving cycle times, dock management, offsite consolidation, food safety and hygiene, and others. ICS logistics usually allocates several docking locations at different levels via links to unload goods to satisfy the various demands from casinos, hotels, MICE, retails, and theaters. ICE logistics also require distributed but space-limited storage space with high warehouse complexity for various storing requirements on wine, dry goods, frozen items, chilled beers, and live seafood. The logistics should focus on leveraging the warehouse inventory between the minimum wastage of goods versus on-time delivery when the order is placed. Operations at ICE docking areas and warehouses are busy and challenging. Short receiving cycle times are expected. Accurate forecast of the incoming traffic of goods and load balancing at docks and warehouses promote lean and efficient ICE logistics.

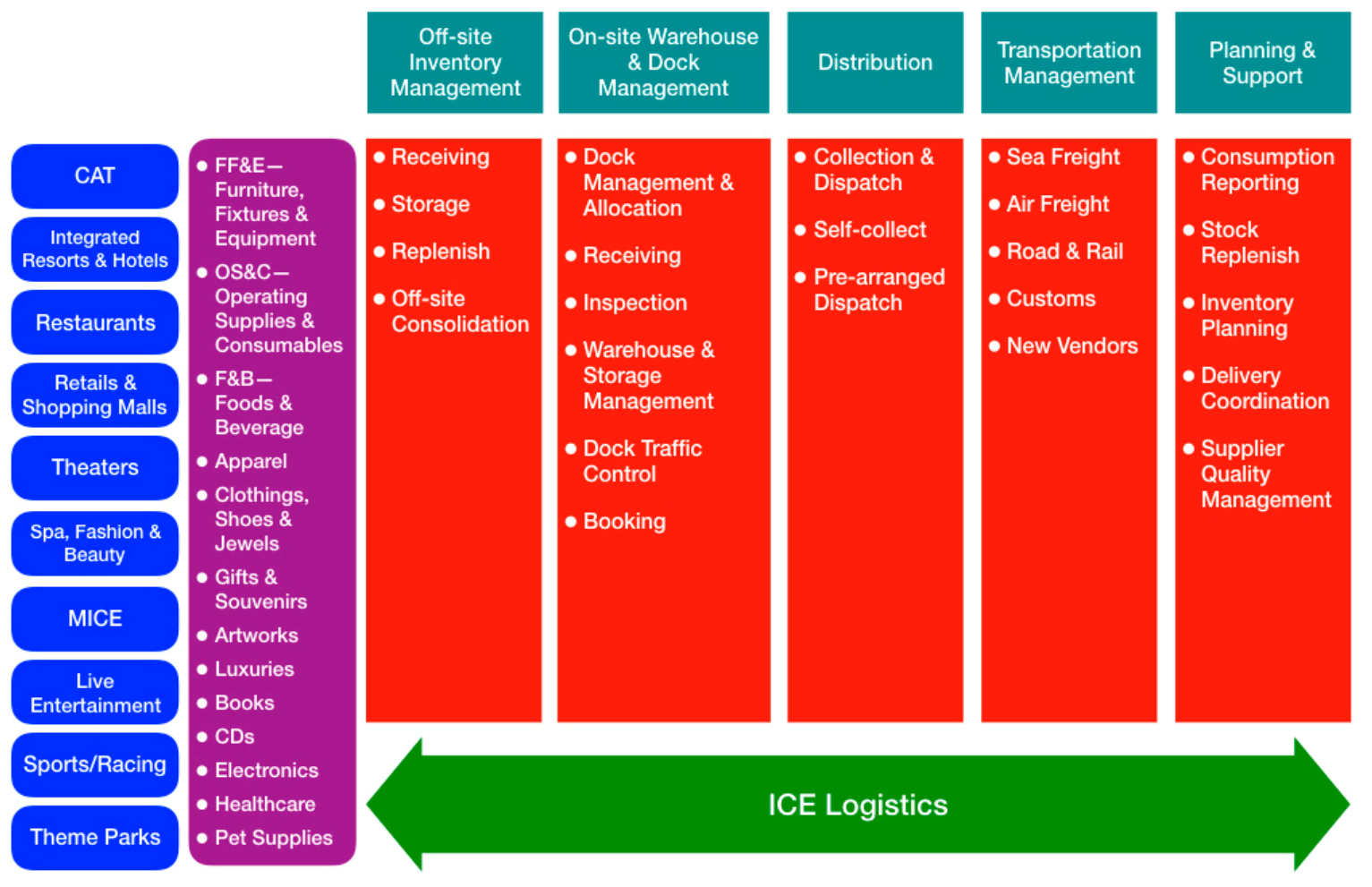

Figure 1. ICE logistics operations.

All the participants in a logistics process are expected to handle goods with their components and materials complying with the requirements and criteria set by clients and by regulations. Material inventories may change from hand to hand. For precious assets or expensive commodities, 
it may be useful to prove and track ownership in their life cycle throughout the supply chain. This is especially critical to comply with auditors' requests to verify asset location and integrity, and if the materials come from areas with environmental social responsibility. Although information about the movement of goods is shared, confidential data, like prices and value of goods, remain protected along the entire process flow in ICE logistics.

The importance of the possession of goods cannot be emphasized too much along the flow of inventories in ICE logistics. The ability to prove the uniqueness of ownership makes this relevant. The ability to track the possession of products and determine their provenance becomes a must for any participant that wants to prove the origin of goods to protect themselves from counterfeiting and to comply with strict health/safety regulations. Completion of added-valued activities to goods like shipping, receiving, and inspecting, may trigger payment requests for the logistics services provided. All these activities should be documented, and some of the information may be shared and made visible to external viewers. However, sensitive data remains strictly confidential to authorized parties only.

Upon shipping or receiving, contractual clauses may demand the declaration or proof of Certificate of Origin (CO) of the goods. The CO documents should be formally created, well validated, and secured with notarization. Moreover, as the ownership of goods changes along the ICE logistics flow, the provenance of all components should be traced back, based on the bills of materials (BOM) of the goods. For third party logistics (3PL) service providers-shipper and freight forwarders-the ICE logistics demand them to be fully regulated and well controlled, which can be achieved by execution of irrevocable and immutable smart contracts of a blockchain. Shipping documents, e.g., bill of loading, advance shipping note, customs clearance papers, should be taken care of, and find the finest repository to different owners.

Dedicated processes for returned goods are needed to ensure that repairs follow well-defined rules and management. The ownership of returned items along their repairing flows should base on warranty, and therefore, change the titles of possession accordingly. In addition to following the defined product or service warranty processes, the repairs and resulting costs should be well managed. Malfunctioning goods may be disassembled and traced back to their origin of components and manufacturers for root-cause analysis and the identification of the source of defects. In ICE logistics, the transactions should be traced and accessed throughout a product's lifecycle.

Inefficiencies in ICE logistics are a major roadblock and the main source of increased costs in ICE industry. The inefficiencies may come from data consistency, operations, and competitiveness, and inequality. It is very inefficient and difficult to trust data between multiple third-party/forth-party logistics providers (3PLs/4PLs) using papers or other limited data formats. Operational inefficiencies manifest from the layers of the middlemen in between. Competitive and inequality inefficiencies come about as a result of centralization and monopolization of relationships.

Timely updates regarding the movement of goods and completion of services save a considerable amount of time, because manual interference is eliminated. ICE logistics expect all transactions to be recordable and trackable with a system that is transparent, efficient, and market-oriented. Blockchain technology is promisingly capable of meeting such expectations by providing the features as below:

- $\quad$ Ownership E Uniqueness

Every document exchanged on the blockchain stores its ownership records with a unique hash code.

- Irreversibility

For every transaction ever made, a certain and verifiable record is kept in each blockchain so that the risk of fraud, abuse, and manipulation of transactions can be minimized.

- Immutability \& Irrevocability

Blockchains provide time-stamps and controls to the provenance of goods. Transaction information such as purchase orders, data and time of arrival, condition of goods, is time-stamped and irrevocable. 
- Notarization

Blockchains are secure, global and decentralized. Storing information on the blockchain provides proof of timestamp and ownership, and is independently verifiable. Under the hood, the document is hashed, and any 3rd party can verify that the document was placed there by the person who holds the private key, like a notary service.

- Provenance

Every product has a story. Blockchains enable every physical product to come with a digital document that proves authenticity and origin, building trust in goods and supply chain.

- Smart Contracts

Smart contracts are stored within the blockchain and can be automatically activated if certain conditions are met. It can offer the perfect mixture of security and ease of use whenever exchanging anything of monetary value.

\section{Concentric Value Circles Model for Blockchain in ICE Logistics Management}

A Concentric Value Circles (CVC) model [35] is a group of concentric value circles. A value circle is defined as the collection of values that directly contribute to the values in its next small concentric value circle. Development of a CVC model begins by identifying the ultimate value of the business, on the basis of which we create an innermost value circle to represent that ultimate value. Any value that directly contributes to this ultimate value is identified and added in a larger value circle, the seconder value circle which is concentric to the innermost value circle. A third concentric value circle is built and each of its values directly contribute to one or more values in the second concentric value circle. The development of the CVC model continues by adding more concentric value circles to the model. Each feature in an outer CVC value circle contributes directly to one or more values in its next inner concentric value circle. For a CVC model with $n$ circles, we denote it as an $n$-circle CVC model.

Let $\mathbf{C}=\left\{\mathrm{C}_{1}, \mathrm{C}_{2}, \ldots, \mathrm{C}_{n}\right\}$ denote a set of all circles in an $n$-circle CVC model. Let $\mathbf{S}_{m}$ denote a set of all values in circle $\mathbf{C}_{m} \in \mathbf{C}$. For value $v \in \mathbf{S}_{m}$ and $m \in\{1,2, \ldots, n-1\}$, define $\sigma(v) \in \mathbf{S}_{m+1}$ be the set of all the values which directly contribute to value $v$. An $n$-circle CVC model, $\left\{\mathbf{S}_{1}, \mathbf{S}_{2}, \ldots, \mathbf{S}_{n}\right\}$, can be constructed through the following Algorithm 1:

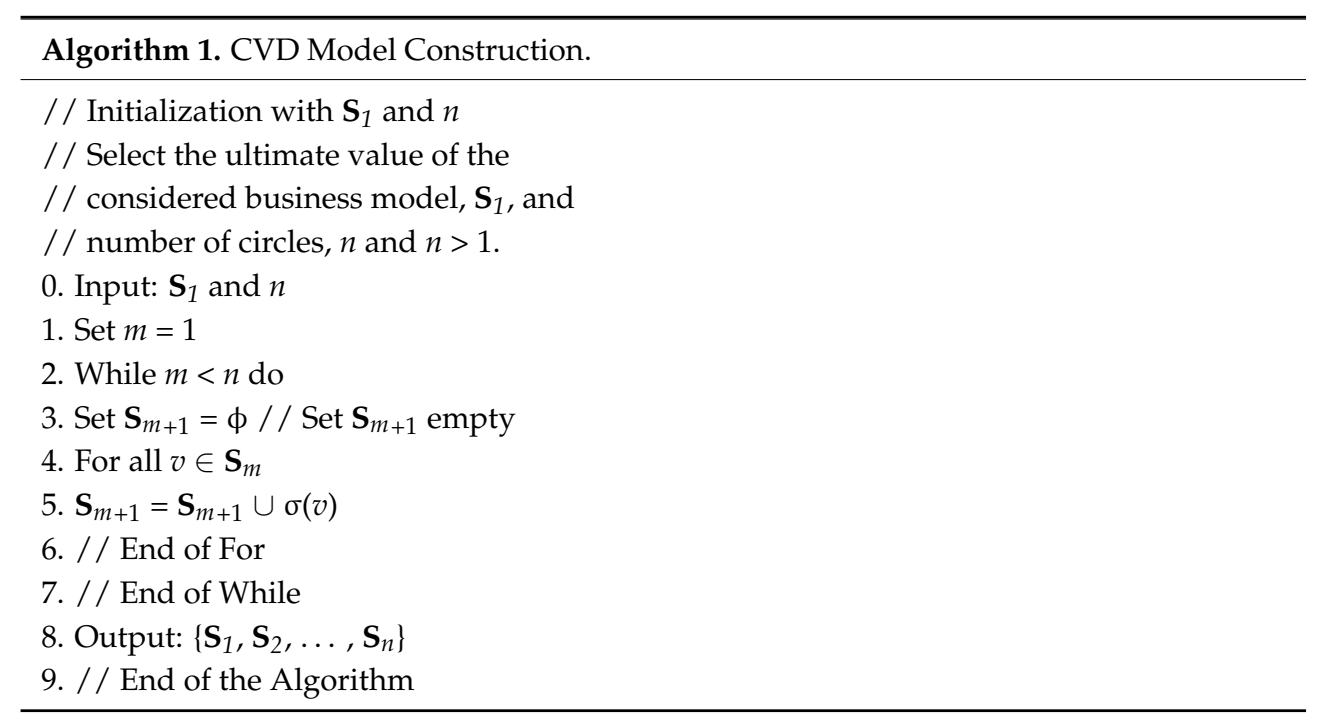

Note that the two input values of the ultimate value of the considered business model, $\mathbf{S}_{1}$, and number of circles, $n$, have to be initialized before the construction of the $n$-circle CVC model. Figure 2 depicts the example of a 4-circle CVC model, where an arrow from value V1 pointing to value $\mathrm{V} 2$ indicates the direct contribution of $\mathrm{V} 1$ to $\mathrm{V} 2$. 


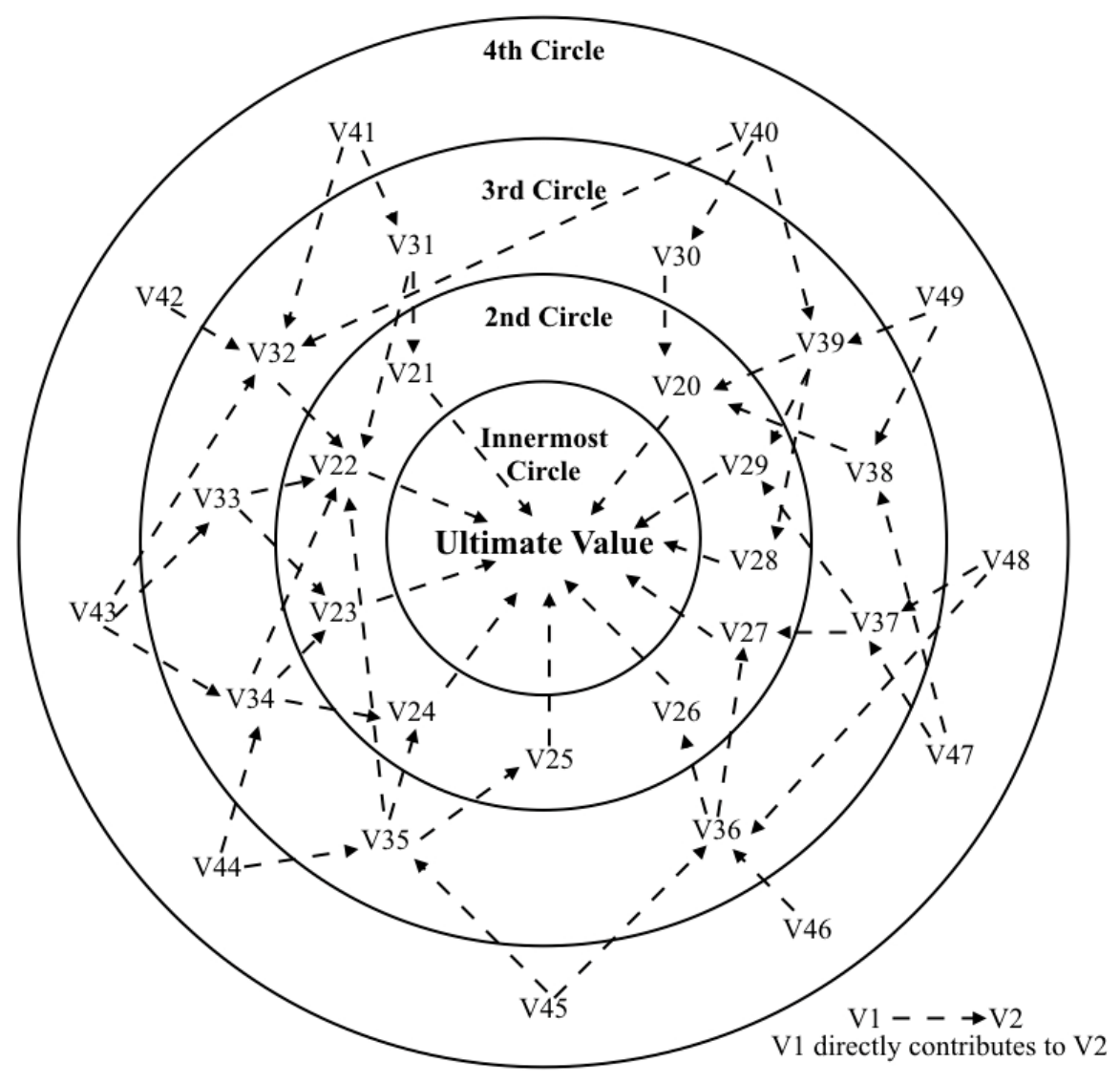

Figure 2. An example of a 4-circle CVC model.

Our ICE CVC model for a blockchain in ICE logistics is created as detailed below. Tourists and customers spending their time and money in ICE are looking for fun-the ultimate value of ICE, gaming, entertainment, hospitality or tourism industry. The innermost concentric value circle of the ICE CVC model is, therefore, fun. In order to directly contribute to the ultimate value of ICE, i.e., fun, six aspects from relaxed, fair, safe, convenient, cost-effective, to friendly are identified and defined in the second concentric value circle. These values are important and fundamental for customers to enjoy funs in ICE.

To contribute these values in the second concentric value circle, values in the third concentric value circle are identified - they are leisure, entertainment, gaming, lottery, sports, racing, security, governance, surveillance, virtual office, door-to-door service, dining, shopping, one-stop service, social, natural, healthy, clean, and themed tour. And a fourth concentric value circle is created to contribute the values in the third value circle directly. Values in the forth value circle are hotel logistics, domestic logistics, global logistics, emergency logistics, fairs $\mathcal{E}$ exhibitions logistics, FEB logistics, retail logistics, and green logistics. The fifth value circle and the outermost circle of our ICE CVC model represents the values to customers in casinos and entertainment. The values are created from blockchains, augmented and virtual reality, IoT, massive connection, end-to-end tracking, robotics $\mathcal{E}$ automation, smart transportation, driverless vehicles, wireless clouds, 3D holograms, X-as-a-Service, and small cells. Figure 3 depicts the proposed ICE CVC model for blockchain in ICE logistics. 


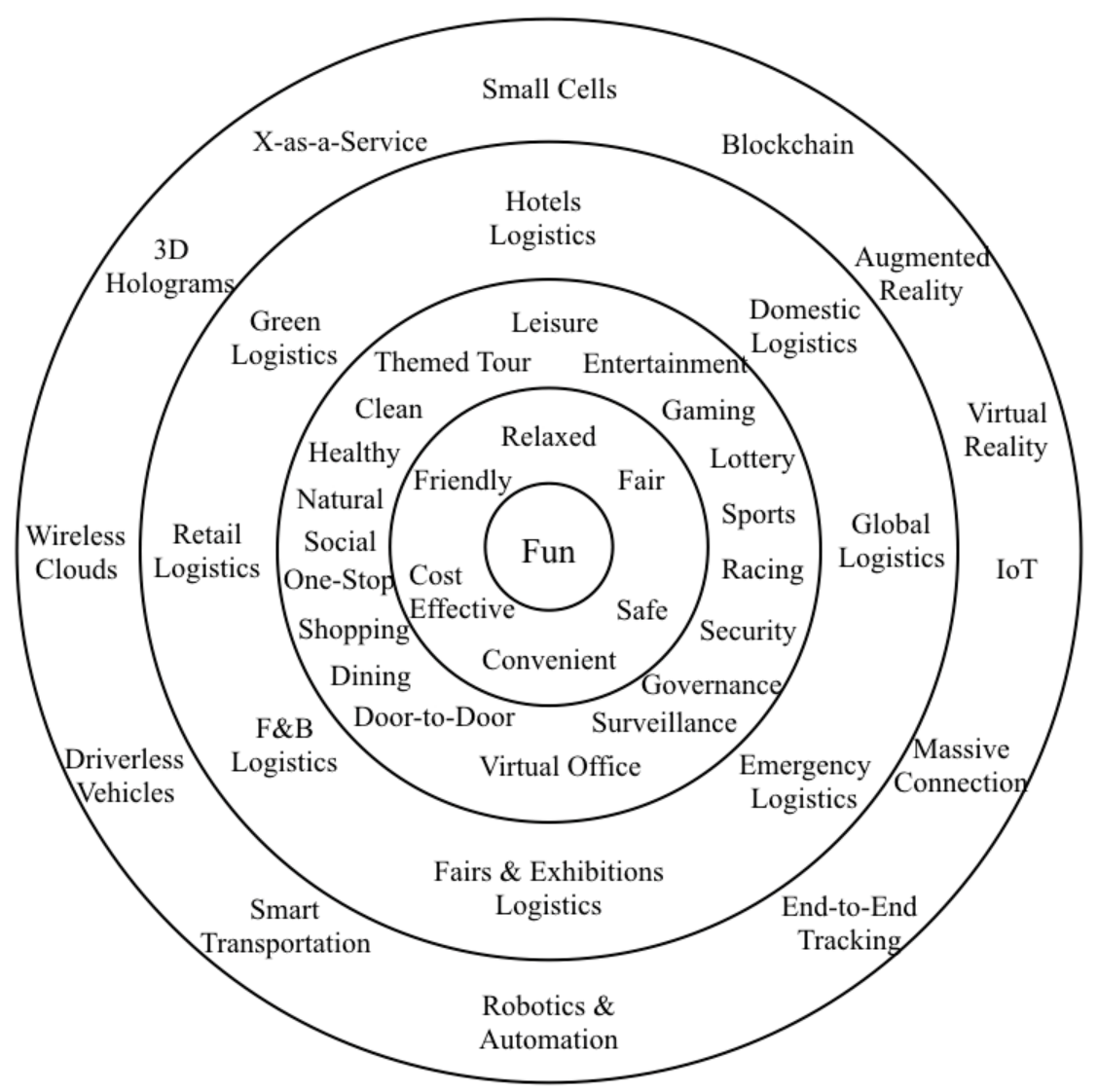

Figure 3. ICE CVC model for blockchain in ICE logistics.

\section{Blockchain-Integrated ICE Logistics Management}

Based on the developed ICE CVC model, a whole new approach, integrated by blockchain logic, could bring the about a transformation of hotels, domestics, foreign-made, emergency, fairs \& exhibitions, F\&B, retails, and green logistics in ICE. Placing the blockchain processes on the new platform would have a very clear objective: to offer ICE customers funs-with relaxed, fair, safe, convenient, cost-effective, and friendly experiences. We develop an open, automated, and transparent platform, TransICE, which is based on blockchain technology to enhance trust mechanisms. In the ICE logistics platform, transporters of air, sea, road or rail, need to understand when goods can be picked up, shipped, and delivered without violating contractual terms of their customers; warehouses and docks need to understand when to receive goods and how to distribute to their customers; exporters and importers need to be able to apply for credit and need to track the status of a transaction at any point in time; and the customers need full transparency to what they buy and use. This platform aims to implement transparency in ICE logistics among the involved parties in real time, and therefore, to provide trustless, safe, and efficient transactions with low trading and operational risks.

\subsection{TransICE: A Blockchain-Integrated ICE Logistics Platform}

The proposed TransICE platform adopts smart contracts for blockchain-integrated ICE logistics [36] and the Hawk model [37], a decentralized smart contract system that stores no financial transactions on the blockchain to keep the privacy of transactions in the public. The TransICE platform is organized into three layers-the Data layer at the bottom, the Smart Contracts layer in the middle, and the Interface layer on the top, as shown in Figure 4. Five constituting modules, blockchain, consensus protocols, role, transactions, and privacy laws, comprise the Data layer as the fundamentals of TransICE. Above the Data layer is the Smart Contracts layer. A smart contract [19], residing at a specific address on a blockchain, is a combination of code snippets (the functions) and data (the states). 
In blockchain technology, Ethereum [38] supports user-defined and Turing complete state machines. Once deployed, the smart contract is executed on all Ethereum nodes as a replicated state machine. The TransICE software stack of smart contracts follows the same design for a typical Ethereum node. For a fully validated Ethereum node, the smart contract contains the entire history of the blockchain. Furthermore, for a non-validating Ethereum node, the smart contract stores only the block headers. Beside the shared states of the blockchains (crypto-value, for example), each smart contract has access to its own states. Figure 5 presents the blockchain software stack on a typical Ethereum node [39]. Smart contract states are maintained as well as normal transactions. Each smart contract can be identified by a unique address which has its own data (the state). Upon receiving a transaction request to its address, the code residing at the smart contract executes automatically by Ethereum Virtual Machine (EVM), the runtime environment for smart contracts. In Figure 5, the dashed line indicates that a smart contract can even assess to other smart contract(s) in the blockchain.

Eight types of smart contracts are defined in TransICE for operations in ICE logistics. They are receiving, shipping, inspection, settlement, dock scheduling, warehouse storage, distribution, and customs. Atop the ICE software stacks, the Interface layer is made of services and applications, including ICE logistics service, 3PL/4PL (third-parties logistics/forth-parties logistics) service, ICE applications service, ICE customer service, trading finance service, sustainability service, supplier/source management, quality management, and events management applications.

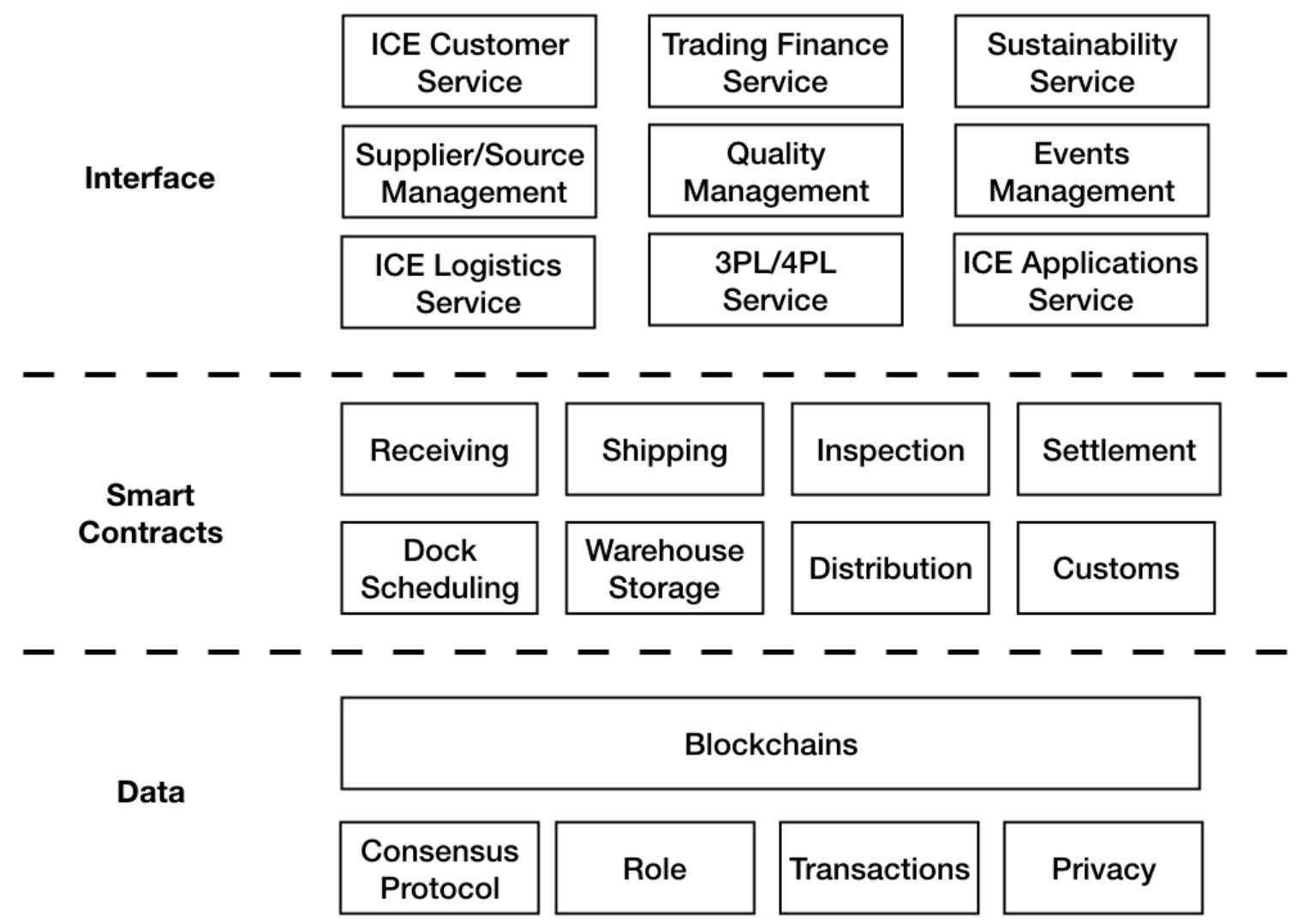

Figure 4. TransICE software structure. 


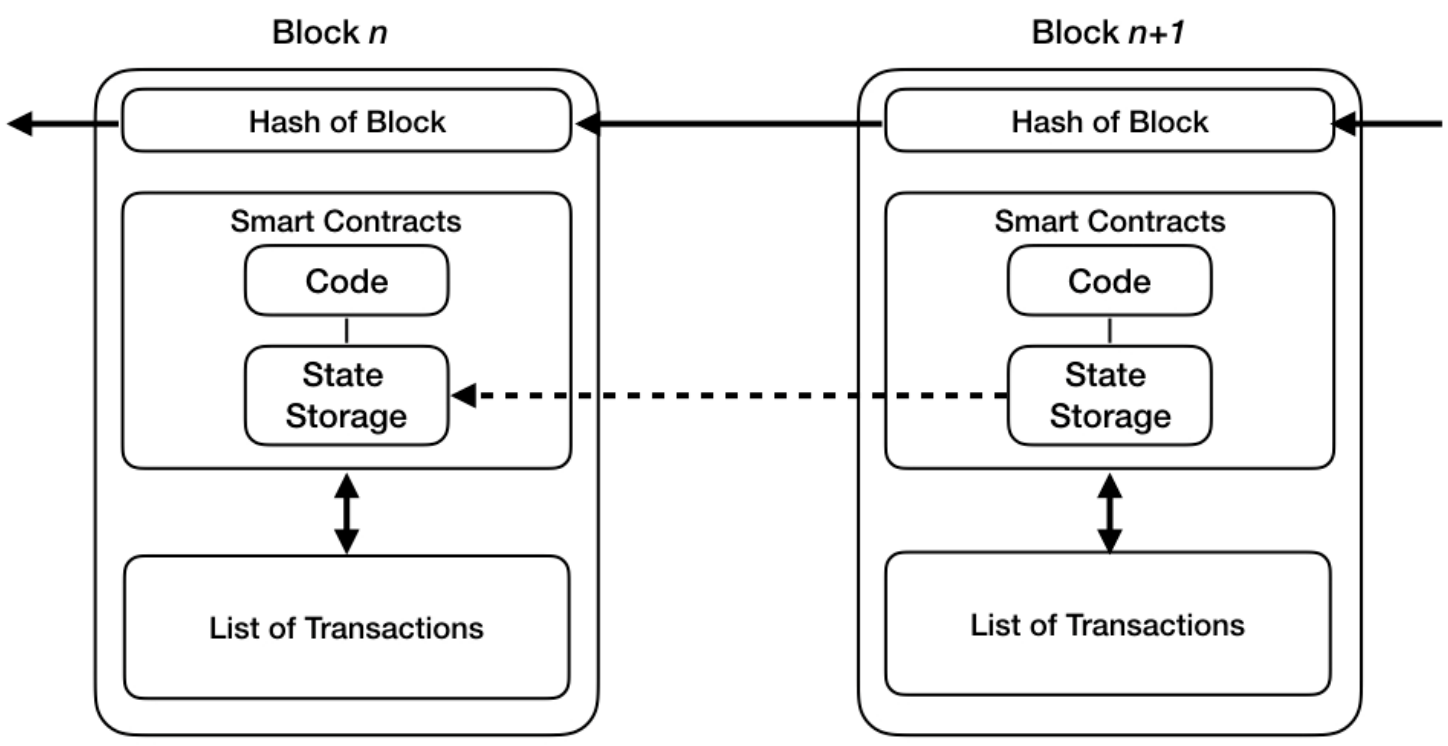

Figure 5. Blockchain software stack on an Etherem node.

\subsection{Hawk-Based Smart Contracts}

Blockchains in the Hawk model are considered to be a group of decentralized mining processes that follow a distributed, secure, and consensus protocol based on the global state. Blockchain technology is designed to be a conceptual trusted ledger. And the trust of the ledger is for correctness and availability only, not for privacy. In Hawk, a blockchain holds a global ledger for every pseudonym of the participants, and also executes user-specific snippets of code. A formal model of the blockchain is defined in Hawk. It consists of ideal specifications and code snippets of the protocol that will be executed by all consensus nodes. Common features such as time, global ledger, pseudonyms, and messages, which are basic to smart contract applications, are designed as wrappers in the Hawk model. The manager has a special role, i.e., to facilitate execution of programs in Hawk. The manager can read users' private data and is trusted not to disclose the data, even though the manager is not a trusted third party as in conventional transactions, and is unable to alter the execution in Hawk. When applying the Hawk model to ICE logistics, the manager is the customer (C), like the functions of Procurement \& Supply Chain in the ICE. The role of users can be the suppliers (S) of goods like farmers and manufacturers, the logistics providers $(L)$ like shippers and transporters, the warehouses $(W)$, or the docks $(D)$ in the ICE.

A Hawk program $\phi$ is composed of two parts-the public part of $\phi_{\text {pub }}$ and the private part of $\phi_{\text {priv }}$. The public part $\phi_{\text {pub }}$ refers, manipulates, or uses no private data and money, while the private part $\phi_{\text {priv }}$ handles private data, like proposed amount of the deal, as well as the ordered quantity. $\phi_{\text {pub }}$ computes and settles the transactions and payment amongst the parties.

A Hawk program is composed of three snippets of code-the ideal snippet, IdealP, the blockchain snippet, BlockchainP, and the user snippet, UserP, that jointly define the cryptographic protocol between the blockchain, users, and the manager. The ideal snippet, IdealP, defines the requirements of correctness and security with a specification under the assumption of existence of a fully trusted party. IdealP utilizes a wrapper to support exact implementation of execution semantics. Two specifications, one for private ledger and currency transfer and the other for special primitives, are implemented in Hawk. Once a private ledger is created and identified in Hawk, three special primitives of Freeze, Compute, and Finalize are used to enable transactional privacy and programmability at the same time. Based on the existence of a private ledger, Hawk supports private currency and defines an ideal protocol, Ideal $P_{\text {cash }}$ to describe the requirements of a private ledger.

The public ledger, ledger, is used by both the ideal program, IdealP, and the blockchain program, BlockchainP. Only when either IdealP or BlockchainP updates ledger, money transfers can be committed. 
The ideal protocol IdealP cash specifies both the requirements of a private ledger and the corresponding currency transfer. Two currency transfer operations, Mint and Pour, are defined as below:

- Mint User $U$ uses Mint to transfer money from public ledger ledger to user's private pool, denoted as Coins $[U]$. For each user, a money transfer will create a private point associated with a value val.

- Pour A user uses Pour to spend money in his (or her) private pool privately.

Consider the ideal case. When an honest user $U$ executes a Mint operation, the adversary $A$ learns the minting and the pair $(U, v a l)$ that coins are being moved from public ledger ledger to user's private pool. Note that operations on the public pool are observable to $A$. However, when an honest user $U$ executes a Pour operation, the adversary $A$ is aware of only the outputs from pseudonyms $U_{1}$ and $U_{2}$. Neither the name of the spender nor the amount of coins in the private pool Coins being spent can be observed. The spent coins are anonymous in the private pool Coins. Note that, to further enhance anonymity, a fake of the pseudonym, either $U_{1}$ or $U_{2}$, is generated on the fly, and only the real user receives the pour.

In order to enable transactional programmability and privacy simultaneously, there are three primitive modules-Freeze, Compute and Finalize, defined in the formal specifications of the ideal program IdealP $P_{\text {hawk. }}$. In the Hawk protocol suite, the Freeze module lets parties commit not only to normal data, but also coins. Committed coins are frozen in the contract. During Compute, parties open their committed data and currency to the manager, so that the manager can compute. Based on the outcome, the manager constructs new private coins to be paid to each recipient. Then the manager submits to the blockchain both the new private coins as well as zero-knowledge proofs. The previously frozen coins are now reallocated and finalized among the users. Three essential primitives are defined as follows:

- $\quad$ Freeze. Party P requests $I_{d e a l P}$ hawk to move one coin from its private pool Coins to FrozenCoins. The coin is first frozen in the blockchain. The private input of Party $P$, denoted as in, is recorded in FrozenCoins. Upon the request and before moving forward with the Freeze function, Ideal $P_{\text {hawk }}$ checks whether Party $P$ has not called Freeze before or whether there is a coin $(P$, val $)$ in Coins.

- Compute. Once Party $P$ moves forward with the Compute function, both the private input of Party $P$, in, and its frozen coin value, val, become disclosed to the manager $P_{M}$.

- Finalize. IdealP hawk computes the outcome of $\phi_{\text {priv }}$ on all parties' inputs and corresponding frozen coin values, after receiving a public input valM from the manager $P_{M}$. Ideal $P_{\text {hawk }}$ also reallocates the FrozenCoins based on the outcome of $\phi_{\text {priv }}$ IdealP hawk maintains the conservation of money by ensuring the total of FrozenCoins equals to all the output coins. IdealP hawk is a function of the public Hawk contract $\phi_{p u b}$ whose purpose is to assert the wellformedness of the input of the manager, in $M$, and reallocate to public deposits.

\subsection{Design of Smart Contracts in TransICE}

The TransICE platform is designed to comply with the public verifiability of provenance data required by the Product Traceability Initiative [40], an initiative designed to help the industry maximize the effectiveness of traceback procedures and develop standards to enhance traceability. Information of product descriptions, locations, handling requirements, and shipment tracking are all public to each actor in the ICE logistics. Provenance of products provides more transparency in ICE logistics where data can be accessed and verified by all actors. However, we adopt public permissioned blockchain [41] technology to protect business critical information from different actors-e.g., price offers and negotiation transactions, against their competitors.

In TransICE, let coin be the unit of cryptocurrency in blockchain-integrated ICE logistics. TransICE utilizes blockchain technology to develop and integrate many useful mechanisms. A blockchain in TransICE is considered to be a database where all ICE logistics processes are stored and 
processed in form of transactions. It also serves as the logging database where all the logs of auditing functions, forgery of coins, transactions integrity checks, and possible corruption of participants are stored in the blockchain. Furthermore, the design and implementation of TransICE protocols should be lightweight so that they can be embedded as an integral part of the paradigm for Internet of Things (IoT) applications.

Notations are then defined as follows for design and development of the proposed TransICE platform:

C: customer;

W: warehouse;

$D$ : dock;

$S$ : supplier;

$L:$ logistics provider;

$A$ : participant, $A \in\{C, W, D, S, L\}$;

$U$ : user, $U \in\{W, D, S, L\}$;

$\operatorname{bnd}($.$) : a blinding function;$

$\operatorname{dpt}($.$) : a decrypting function;$

ept(.): an encrypting function;

$h s h($.$) : a SHA-256 hash function;$

$r c p($.): a receipt generator function;

rnd(.): a random number generator function;

$\operatorname{sqn}($.): a sequence number generator function;

pair of public and private keys of $\in\{C, W, D, S, L\},=$ (.public, .private);

$\Xi(m)$ : signature of coded message $m$ and private key;

$\delta(m, \Xi)$ : verification of coded signature $\Xi$, message $m$ and public key;

TransICE has developed several protocols executed by the blockchain, the users (suppliers, logistics providers, warehouses, docks), and the manager (customer), as programs written in pseudo code. As an illustrating example of pseudo code of protocols, protocol Algorithm 2 Registration is listed as follows: 


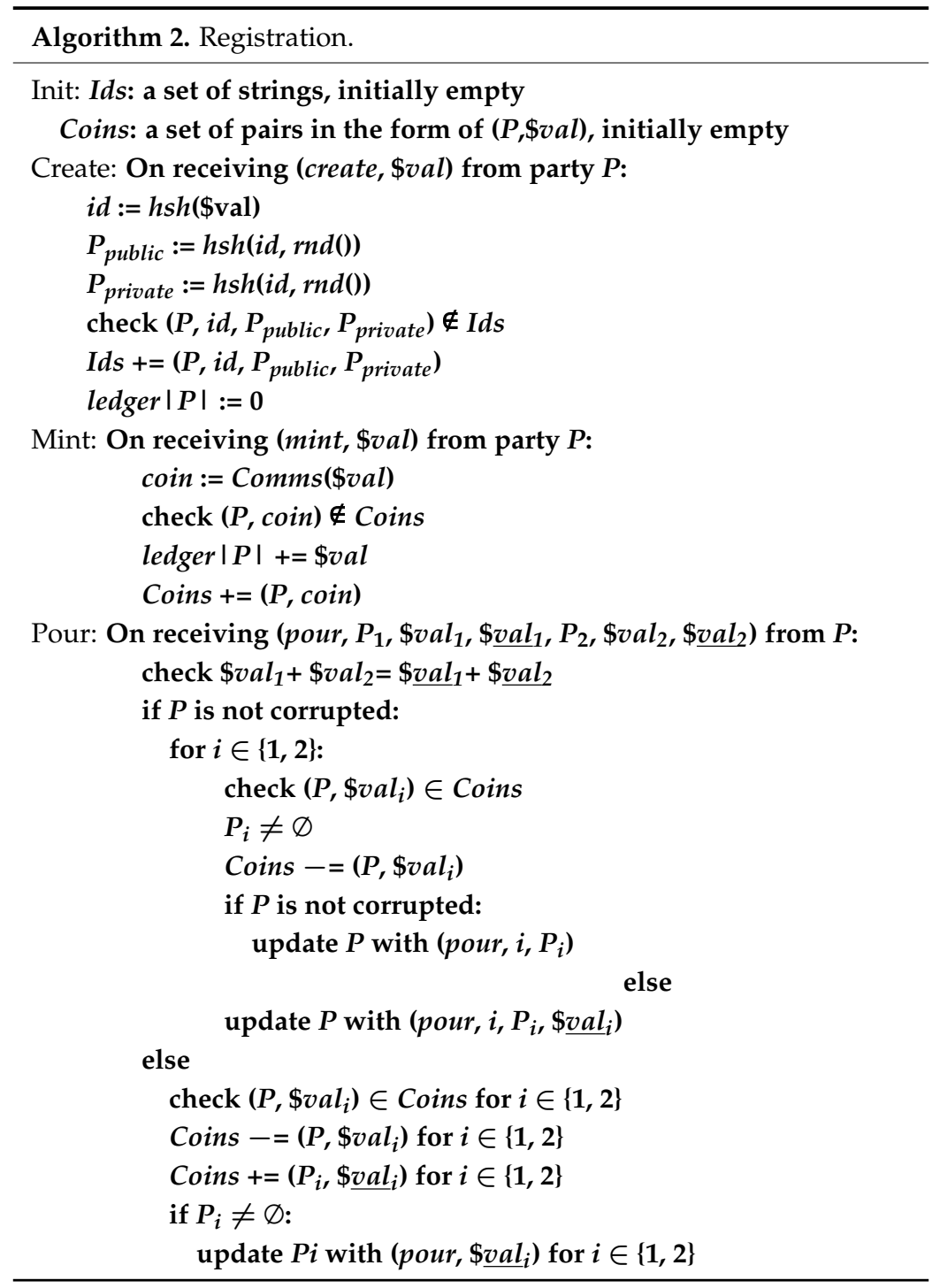

Another illustrating example, protocol Algorithm 3 Pricing, is listed as follows: 


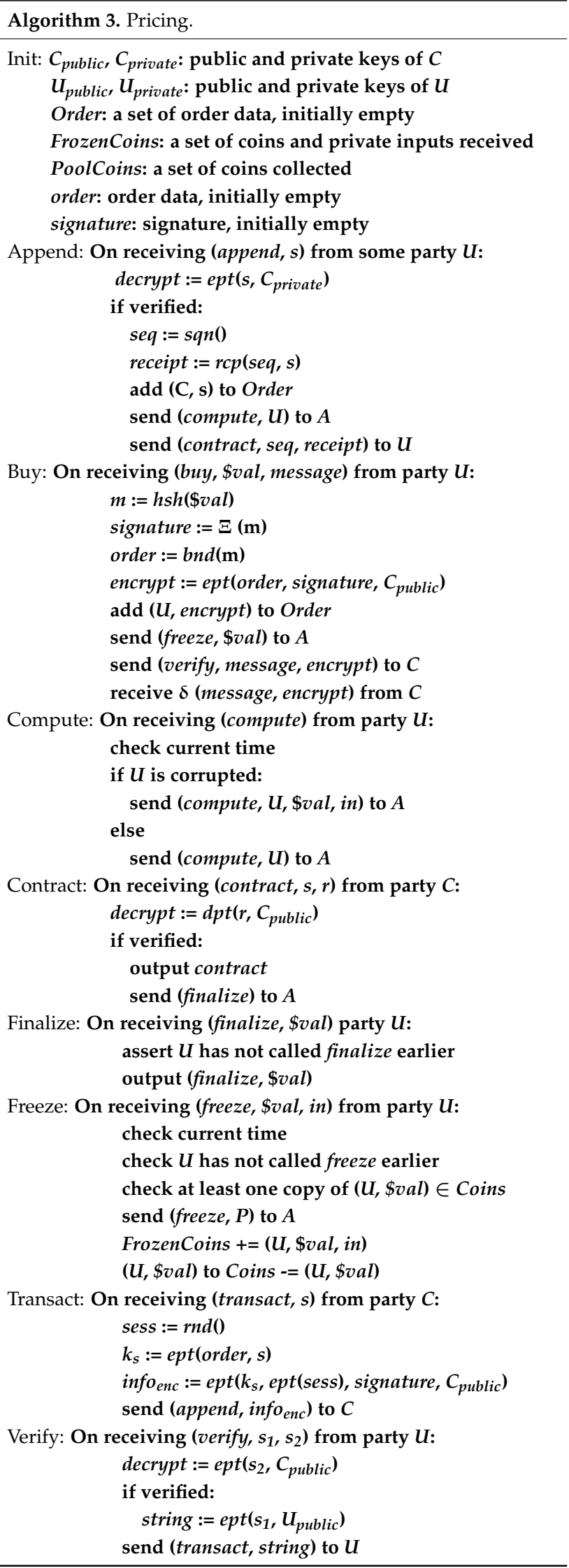




\section{Case Studies}

This Section studies two representative cases in ICE logistics-(1) the Shipment Pricing and Scheduling process; and (2) the Pickup, Shipping and Delivery process. Both the illustrating cases are studied with the Registration and Pricing protocols developed in Section 5 to demonstrate the applications and feasibility of the proposed TransICE platform that uses blockchain technology in ICE logistics.

\subsection{Shipment Pricing and Scheduling in TransICE}

In TransICE, a Shipment Pricing and Scheduling process starts as the supplier (S) uses the Create(), Mint(), and Pour() functions in the Registration protocol and create a new Contract in the INQUIRY state. The Contract also defines specifications and requirements of temperatures and handling of the shipment, deadlines, and penalties for tardiness and violations. A logistics provider $(P)$ uses the $B u y()$ function of the Pricing protocol and makes a price offer to the Contract. The supplier $\mathrm{S}$ uses the Compute() and Append() functions of the Pricing protocol, revises the PRICE, and proposes a counter offer to logistics provider $P$. After receiving the price acceptance from $P ., S$ uses the Freeze() function to close the bidding and provides a list of pickup windows to $P$. $P$ selects a pickup window and sends to $S$. Then, $S$ confirms the pickup window after using the Verify() function of the Pricing protocol. Similar process for delivery to the Customer (C) follows. The supplier (S) uses the Contract() function to send the Contract in the ISSUED state to the logistics providers $(P)$ and executes the Finalize() function to close the Shipment Pricing and Scheduling process. The shipping Contract is also sent to the Customer $(C)$.

Note that, except for the Transact() function, the Shipment Pricing and Scheduling process involves all the functions developed in the Registration and Pricing protocols in Section 5. This case illustrates the application and feasibility of the proposed blockchain-integrated TransICE platform. Figure 6 shows the sequence diagram of the Shipment Pricing and Scheduling process in TransICE.

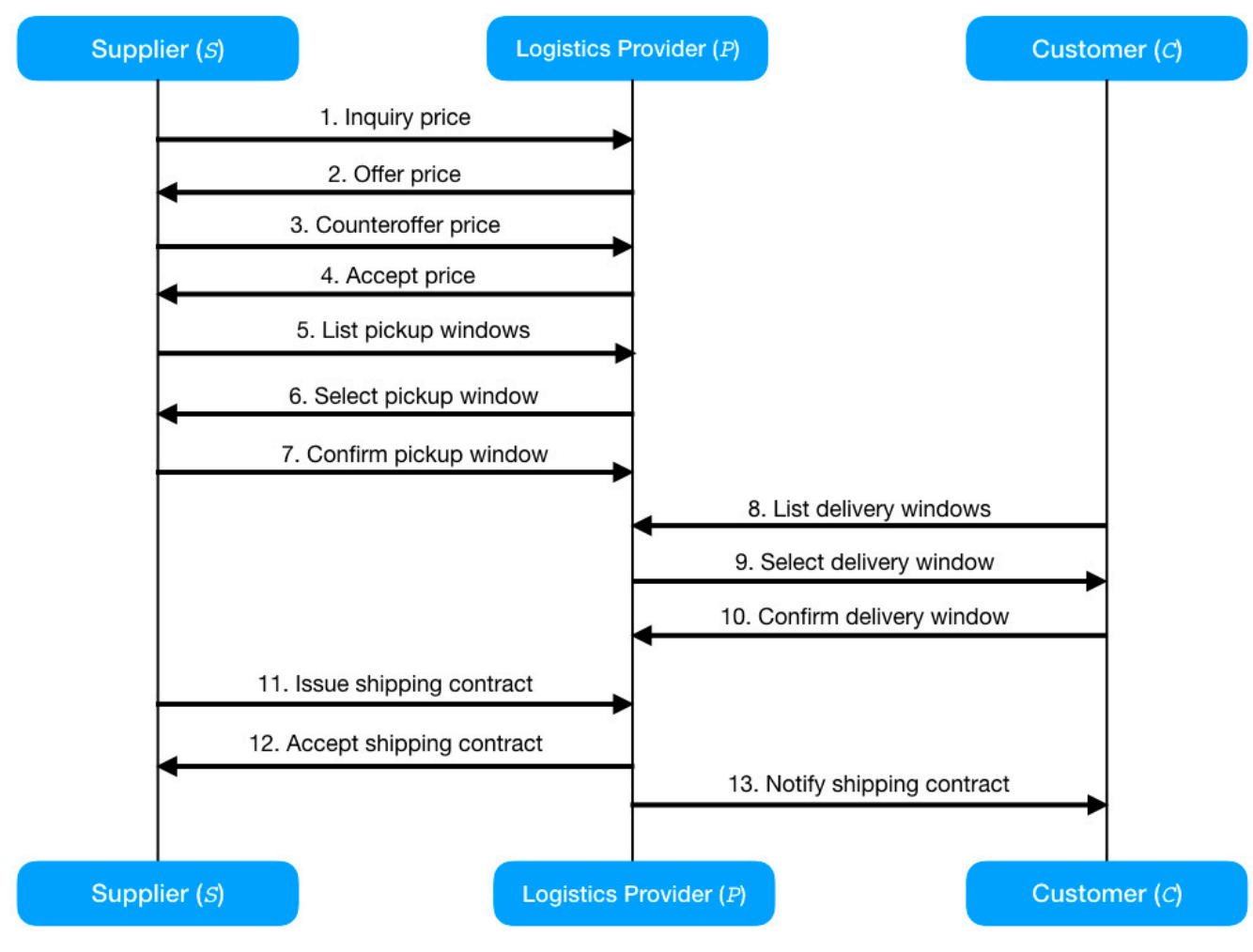

Figure 6. Shipment pricing and scheduling in TransICE. 


\subsection{Pickup, Shipping and Delivery in TransICE}

In TransICE, a Pickup, Shipping and Delivery process starts when the state of a Contract becomes READY_FOR_PICKUP. The logistics provider $(P)$ generates a PICKUP_NOTICE to the supplier $(S)$ once $P$ heads to $S$. After completing loading at $S, P$ uses the Transact() function, sends a PICKUP_SIGNATURE to $S$, and then receives a HANDOFF_SIGNATURE from $S$. During transport, $P$ reports and updates the estimated time of delivery as well as the transportation conditions like temperatures, which are recorded periodically to the blockchain in TransICE. At the same time, the dock $(D)$ requests storage space at the warehouse (W). W uses the Create(), Mint() and Pour() functions of the Registration protocol, create a new Inventory in the INCOMING state, and allocates and confirms the Bin_Location to D. Upon arrival to $D, P$ notifies the arrival to $D$ and records that $P$ is ready to unload with the ARRIVED state. After unloading has finished, logistics provider $P$ sends a DROPOFF_SIGNATURE to dock $D$ and then receives a HANDOFF_SIGNATURE from $D$ if inspection of the goods received complies with the specifications and requirements defined in the Contract. The Contract then enters the DELIVERED state. $P$ notices to $S$ that the shipment is COMPLETED and calls the Finalize() function to close the Pickup, Shipping and Delivery process. At almost the same time, $D$ notifies $W$ to start to place Inventory at the planned Bin_Location. Inventory enters the INVENTORY state with a receiving timestamp recorded in its corresponding blockchain.

Note that in this case, the process involves the Transact() function of the Pricing protocol. In addition, the Shipping and Delivery process in TransICE uses the Finalize() function of the Pricing protocol; and the Create(), Mint() and Pour() functions of the Registration protocol. This case demonstrates the applications and feasibility of the proposed TransICE platform that integrates blockchain technology to ICE logistics management. Figure 7 illustrates the sequence diagram of the Pickup, Shipping and Delivery process in TransICE.

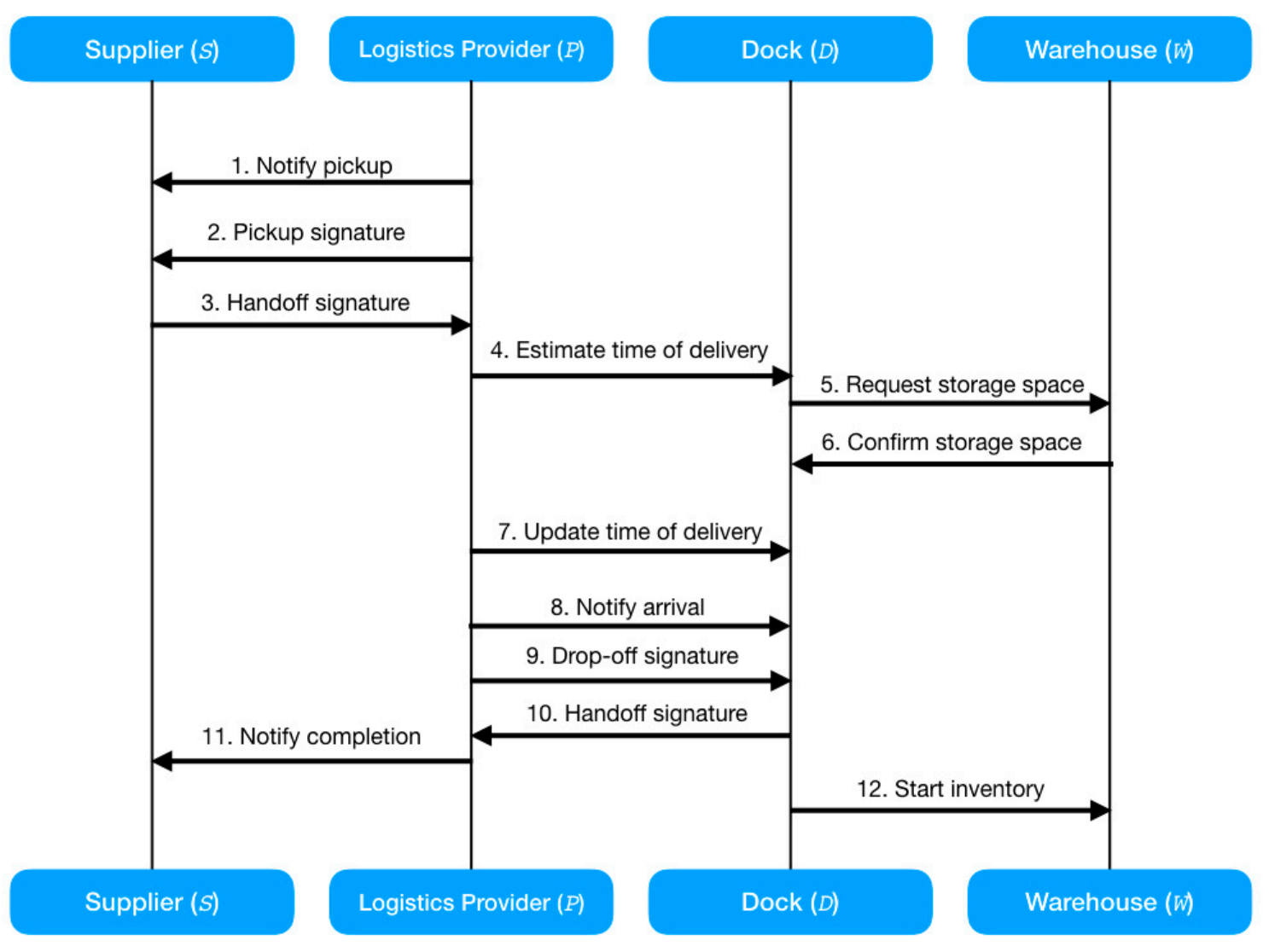

Figure 7. Pickup, shipping and delivery in TransICE. 


\section{Conclusions}

Integrated Casinos and Entertainment (ICE) boosts logistics across all sectors, from lodging, foods and beverages, entertainments, transportation, banking, to government and healthcare, involving several thousands of rooms, tens of F\&B outlets and restaurants, dozens of retail shops and malls, casinos with hundreds of tables and thousands of slots, theaters and museums, event plaza, and exhibition and meeting space of millions square feet to accommodate tens of thousands of participants. ICE logistics deal with supply chains with various stages, in geographically-distributed locations, and with limited and complex storage and warehouses. Challenges in ICE logistics can occur in inbound traffic, distributed and limited storage, warehouse complexity, receiving cycle times, dock management, offsite consolidation, food safety and hygiene, and others. Blockchains, as a means of ensuring transparency and security, can improve the flow of goods and the flow of information in ICE logistics. With blockchains, public availability gives the opportunity to track goods and services from the place of origin to the end customer. The decentralized structure of blockchain gives the ability for participation of all parties in the ICE logistics.

This paper adopts a Concentric Value Circles (CVC) model to analyze and identify the requirements and business opportunities of applying blockchain technology in ICE logistics. Based on the function of smart contracts in blockchain technology, we have developed an open, automated, and transparent platform, TransICE, which adopts the decentralized Hawk model and stores no financial transactions on the blockchain to hold privacy of transactions publicly. Two illustrating examples of pseudo code of protocols, Registration and Pricing, are provided for the development of smart contracts with the Hawk model. Two representative cases of ICE logistics processes of (1) Shipment Pricing and Scheduling; and (2) Pickup, Shipping and Delivery are studied to demonstrate the applications and feasibility of the proposed TransICE platform and the developed smart contracts of the Hawk model.

In order to focus on the development of the TransICE platform, all the processes presented in this paper are based on main scenarios that describe the simple flows through use cases where everything goes right, without difficulty. In real world, there are always exceptions. Exception handling, like return merchandise authorization (RMA) or tardy penalty, is a part of the ICE logistics processes and can be included in the proposed TransICE platform. Development of novel technology cannot guarantee that the derived application will be used, and that it will succeed. Theoretical analysis is needed to better understand the underlying benefits and difficulties which may lead ICE industries to adopt blockchain technology for transparency and traceability in ICE logistics. Future research directions include research and development of formal descriptions and analysis of state transitions in the blockchain system of smart contracts. As one of the technologies that bring cryptocurrencies in play, in addition to logistics management in ICE, blockchains can also serve as the basis technology to provide virtual currency (or e-money) as part of the entire ICE ecosystem. Future research directions may also include research and development of blockchain-integrated tokens for Internet-of-Value services in ICE-to digitalize value of assets, send value and pay for transactions, securely and accurately across entire ICE networks.

Author Contributions: Conceptualization, D.L. and X.W.; Methodology, D.L.; Software, D.L.; Validation, D.L.; Formal Analysis, D.L.; Writing-Original Draft Preparation, D.L.; Writing-Review \& Editing, D.L. and X.W.; Visualization, D.L.

Funding: This research received no external funding.

Conflicts of Interest: The authors declare no conflict of interest.

\section{References}

1. Denhardt, R.B.; Denhardt, J.V. The new public service: Serving rather than steering. Public Adm. Rev. 2000, 60, 549-559. [CrossRef] 
2. Porter, M. Competitive Advantage: Creating and Sustaining Superior Performance; Simon and Schuster: New York, NY, USA, 2008; ISBN 1416595848.

3. Kilby, J.; Fox, J.; Lucas, A. Casino Operations Management, 2nd ed.; John Wiley \& Sons: New York, NY, USA, 2005; ISBN 978-0471266327.

4. Ahn, J.; Back, K.J. Integrated resort: A review of research and directions for future study. Int. J. Hosp. Manag. 2018, 69, 94-101. [CrossRef]

5. Christopher, M. Logistics and Supply Chain Management, 5th ed.; Pearson: Harlow, UK, 2016; ISBN 978-1-292-08379-7.

6. Barreto, L.; Amaral, A.; Pereira, T. Industry 4.0 implications in logistics: An overview. Procedia Manuf. 2017, 13, 1245-1252. [CrossRef]

7. Timm, I.J.; Lorig, F. Logistics 4.0: A challenge for simulation. In Proceedings of the 2015 Winter Simulation Conference, Huntington Beach, CA, USA, 6-9 December 2015; IEEE Press: Piscataway, NJ, USA, 2015; pp. 3118-3119.

8. Szymańska, O.; Adamczak, M.; Cyplik, P. Logistics 4.0-A new paradigm or set of known solutions? Res. Logist. Prod. 2017, 7, 299-310. [CrossRef]

9. Marina Bay Sands (MBS). Available online: https://www.marinabaysands.com (accessed on 15 July 2018).

10. Swan, M. Blockchain: Blueprint for A New Economy; O’Reilly: Sebastopol, CA, USA, 2015; ISBN 978-1-491-92049-7.

11. Szabo, N. Formalizing and Securing Relationships on Public Network. First Monday 1997, 2. [CrossRef]

12. Philander, K.S.; Walker, D.M.; William, R. Eadington and the economics of gambling. UNLV Gaming Res. Rev. J. 2012, 16, 9-18.

13. Eadington, $\mathrm{W}$. The spread of casinos and their role in tourism development. In Contemporary Issues in Tourism Development; Routledge: London, UK, 2003; pp. 135-150, ISBN 041520691X.

14. Vong, F. Changes in residents' gambling attitudes and perceived impacts at the fifth anniversary of Macao's gaming deregulation. J. Travel Res. 2009, 47, 388-397. [CrossRef]

15. Singapore Tourism Board. Available online: https://www.stb.gov.sg/statistics-and-market-insights (accessed on 15 July 2018).

16. The Straits Times. Available online: https://www.straitstimes.com/opinion/asia-pacifics-bet-on-casinofuelled-economic-growth (accessed on 15 July 2018).

17. Iansiti, M.; Lakhani, K.R. The truth about blockchain. Harv. Bus. Rev. 2017, 95, 118-127.

18. Nakamoto, S. Bitcoin: A Peer-to-peer Electronic Cash System. Available online: https://bitcoin.org/bitcoin. pdf (accessed on 15 July 2018).

19. Buterin, V. A Next-generation Smart Contract and Decentralized Application Platform. Available online: https:/ / cryptorating.eu/whitepapers/Ethereum/Ethereum_white_paper.pdf (accessed on 15 July 2018).

20. Tapscott, D.; Tapscott, A. Blockchain Revolution: How the Technology behind Bitcoin is Changing Money, Business, and the World; Penguin Random House: New York, NY, USA, 2016; ISBN 9781101980149.

21. Kirkland, R.; Tapscott, D. How blockchains could change the world. McKinsey Q. 2016, 3, 110-113.

22. Li, J.; Greenwood, D.; Kassem, M. Blockchain in the built environment: Analysing current applications and developing an emergent framework. In Proceedings of the Creative Construction Conference 2018, Ljubljana, Slovenia, 30 June-3 July 2018; Diamond Congress Ltd.: Budapest, Hungary, 2018. (in press).

23. Miščević, G.; Tijan, E.; Ražen Žgaljić, D.; Jardas, M. Emerging trends in e-logistics. In Proceedings of the MIPRO 2018 41st International Convention (DEGLGPS), Opatija, Croatia, 21-25 May 2018.

24. Korpela, K.; Hallikas, J.; Dahlberg, T. Digital supply chain transformation toward blockchain integration. In Proceedings of the 50th Hawaii International Conference on System Sciences, Waikoloa, HI, USA, 4-7 January 2017.

25. Gallay, O.; Korpela, K.; Tapio, N.; Nurminen, J.K. A peer-to-peer platform for decentralized logistics. In Proceedings of the Hamburg International Conference of Logistics (HICL), Hamburg, Germany, 12-14 October 2017; Epubli: Hamburg, Germany, 2017; pp. 19-34. [CrossRef]

26. Diveco Logistics. Whitepaper. Available online: https://diveco.net/DIVECO_WHITEPAPER_EN.pdf (accessed on 15 July 2018).

27. Popov, S. The Tangle. Available online: http://tanglereport.com/wp-content/uploads/2018/01/IOTA_ Whitepaper.pdf (accessed on 15 July 2018).

28. QUASA. The Whitepaper. Available online: https:/ / www.quasa.io/whitePaper (accessed on 15 July 2018). 
29. LogisticsX. Whitepaper-The Future of Decentralised Last Mile Logistics. Available online: https: / / logisticsx.io/documents / LogisticsX-Whitepaper-V1.4-11-June-2018.pdf (accessed on 15 July 2018).

30. Liu, S.; Kasturiratne, D.; Moizer, J. A hub-and-spoke model for multi-dimensional integration of green marketing and sustainable supply chain management. Ind. Mark. Manag. 2012, 41, 581-588. [CrossRef]

31. Teece, D.J. Business models, business strategy and innovation. Long Range Plan. 2010, 43, 172-194. [CrossRef]

32. DaSilva, C.; Trkman, P. Business model: What it is and what it is not. Long Range Plan. 2014, 47, 379-389. [CrossRef]

33. Nowiński, W.; Kozma, M. How can blockchain technology disrupt the existing business models? Entrep. Bus. Econ. Rev. 2017, 5, 173-188. [CrossRef]

34. Francisco, K.; Swanson, D. The supply chain has no clothes: Technology adoption of blockchain for supply chain transparency. Logistics 2018, 2, 2. [CrossRef]

35. Liao, D.-Y.; Wang, X.H. 5G Wireless micro operators for integrated casinos and entertainment in smart cities. In Smart Cities; Springer: New York, NY, USA, 2018. (in press)

36. Liao, D.-Y.; Wang, X.H. Design of a blockchain-based lottery system for smart cities applications. In Proceedings of the 2017 IEEE 3rd International Conference on Collaboration and Internet Computing (CIC), San Jose, CA, USA, 15-17 October 2017; pp. 275-282.

37. Kosba, A.; Miller, A.; Shi, E.; Wen, Z.; Papamanthou, C. Hawk: The blockchain model of cryptography and privacy-preserving smart contract. In Proceedings of the 2016 IEEE Symposium on Security and Privacy (SP), San Jose, CA, USA, 23-25 May 2016; pp. 839-858.

38. The Ethereum Project. Available online: https://www.ethereum.org (accessed on 15 July 2018).

39. Dinh, T.; Wang, J.; Chen, G.; Liu, R.; Ooi, B.; Tan, K. BLOCKBENCH: A framework for analyzing private blockchain. In Proceedings of the 2017 ACM International Conference on Management of Data, Chicago, IL, USA, 14-19 May 2017; pp. 1085-1100.

40. The Produce Traceability Initiative. Available online: https://www.producetraceability.org (accessed on 15 July 2018).

41. Wüst, K.; Gervais, A. Do you need a Blockchain? IACR Cryptol. ePrint Arch. 2017, 2017, 375.

(C) 2018 by the authors. Licensee MDPI, Basel, Switzerland. This article is an open access article distributed under the terms and conditions of the Creative Commons Attribution (CC BY) license (http://creativecommons.org/licenses/by/4.0/). 